\title{
3/2 Harmonic Generation - The Clue to the Mechanism of Ultrafast Laser Nanostructuring
}

\author{
Aabid Patel, Mindaugas Gecevičius, Rokas Drevinskas, Martynas Beresna and Peter G. Kazansky \\ Optoelectronics Research Centre, University of Southampton, SO17 1BJ, United Kingdom \\ ap7g12@orc.soton.ac.uk
}

\begin{abstract}
The correlation between $3 / 2$ harmonic generation and self-assembled nanostructuring during ultrafast laser writing in glass was observed with an unexpected enhancement of harmonic generation for elliptically polarized light.

OCIS codes: (140.3390) Laser material processing; (320.7120) Ultrafast phenomena
\end{abstract}

The nanostructuring of transparent media with ultrafast laser pulses has attracted significant interest due to its unique applications. It was demonstrated that above a certain threshold, self-assembled nanogratings in silica glass can be induced [1-4]. Although the mechanism that triggers the nanostructure is still unclear, a theory has been introduced involving the mechanism of nanogratings based on two plasmon parametric decay [5]. A signature of two-plasmon decay is the generation of the $3 / 2$ harmonic, which previously was reported only for high energies [6]. In order to provide strength to the theory for ultrafast nanostructure formation, the generation and characterisation of the $3 / 2$ harmonic at the regimes of nanograting formation are required. Here we present explicit evidence of the two-plasmon process at the laser pulse energies close to ultrafast laser nanostructuring. We demonstrate strong dependence of $3 / 2$ harmonic on the polarization ellipticity of the pump beam, which sheds new light on the interaction of femtosecond light pulses with electron plasma. We also analyse temporal behaviour of $3^{\text {rd }}$ and $2^{\text {nd }}$ harmonics and their polarization dependence.

The experimental set-up used for harmonic generation was composed of a mode-locked regenerative amplified Yb:KGW based femtosecond laser system PHAROS (Light Conversion Ltd.) operating at $\lambda=1030 \mathrm{~nm}$ and delivering pulses of $300 \mathrm{fs}$ at a $200 \mathrm{kHz}$ repetition rate. The laser beam was focused into the bulk of the silica sample via a $\times 10$ (NA $=0.16$ ) aspheric lens. The signal was collected into a fibre by collimating the transmitted light along the line of incidence with a $\times 10(\mathrm{NA}=0.16)$ aspheric lens with an antireflection coating for the visible and focusing with an UV fused silica $f=25.0 \mathrm{~mm}$ lens. Before the sample, a filter was inserted in order to ensure that all harmonics were generated from the sample and not from the optical elements placed before the sample. The spectrum was analysed with an Andor Shamrock SR-500i imaging spectrometer, where two gratings were used to vary the spectral range of the measurement (150 and 1200 lines/mm). A series of spectral measurements for varying pulse energy were taken translating the sample and at stationary positions to determine the power and time dependence of the observed harmonics. The harmonic generation experiments were performed with linearly and circularly polarized pumps. The elliptical polarization was obtained with a zero order quarter-wave plate for $1064 \mathrm{~nm}$. The polarization of emitted harmonics was analysed with a linear polarizer.

(a)

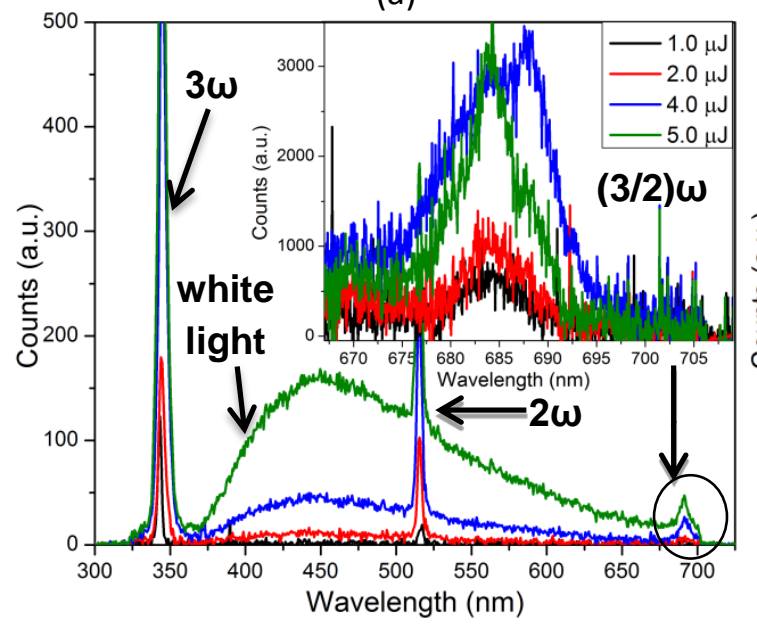

(b)

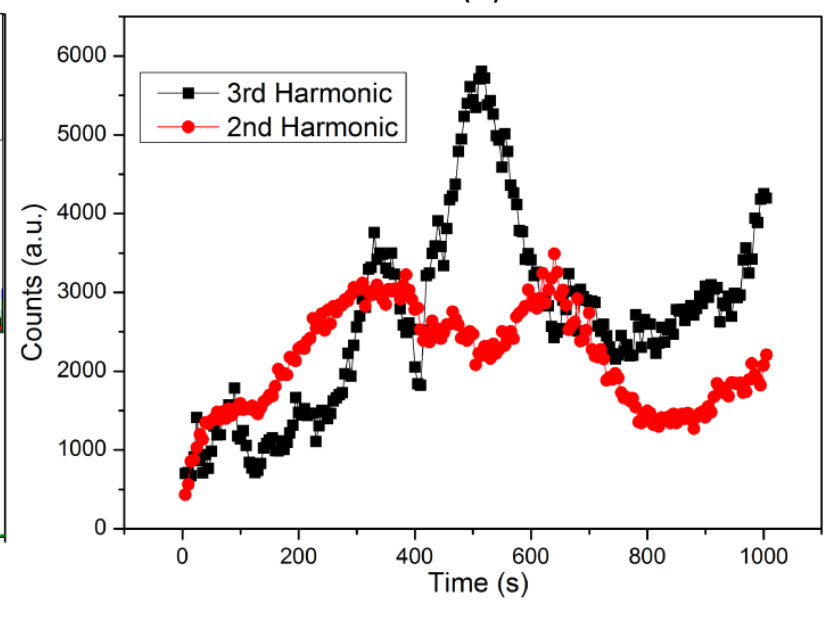

Fig. 1 a) Spectrum of harmonics for varying pulse energies ( $200 \mathrm{kHz}$ repetition rate). White light emission and the double peak structure for $(3 / 2) \omega$ are observed as pulse energy is increased. b) Time dependence $2^{\text {nd }}$ and $3^{\text {rd }}$ harmonic for $0.2 \mu \mathrm{J}$ pulse energy.

The spectrum measured for varying pulse energies of the generated harmonics is measured with 150 lines/mm grating and the (3/2) $\omega$ measurement done with 1200 lines/mm (Figure 1a). Within the measured spectrum, $2^{\text {nd }}$, 
$3^{\text {rd }}$ and $3 / 2$ harmonics are observed with white light emission being more evident as pulse energy increases. All three harmonics increase with pulse energy and evolve on the time scale of several minutes (Figure $1 \mathrm{~b}$ ). The signals of $2^{\text {nd }}$ and $3^{\text {rd }}$ harmonic increase steadily within the first few minutes of measurement and then begin to randomly oscillate. The increase of $2^{\text {nd }}$ harmonic can be attributed to a collection of charges at the point of focus [7]. The time dependence of $3^{\text {rd }}$ harmonic and the initial generated signal increase is currently under investigation.

All three harmonics are polarized in the direction of the pump beam's polarization when tested with the linear polarizer. The $3 / 2$ harmonic is co-polarized with the pump beam and the white light emission is unpolarized.

(a)

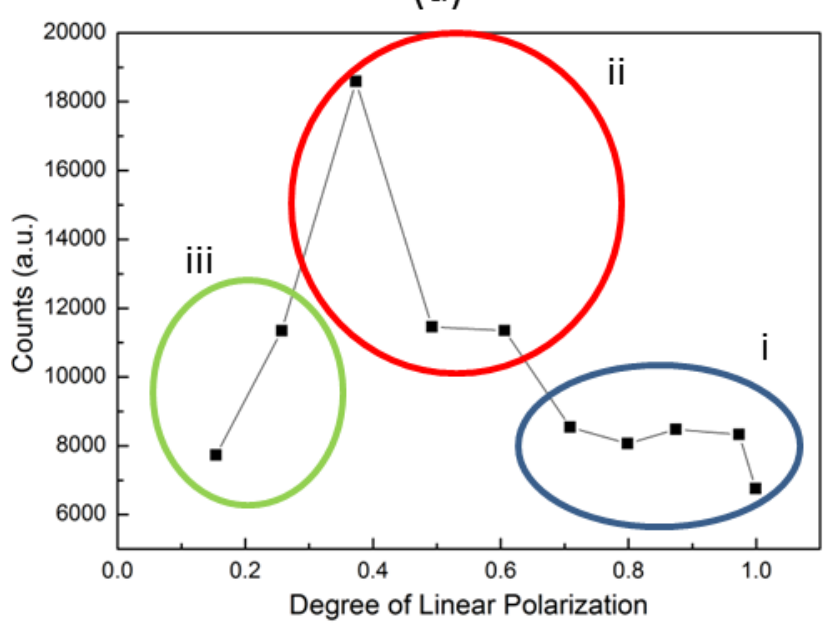

(b)

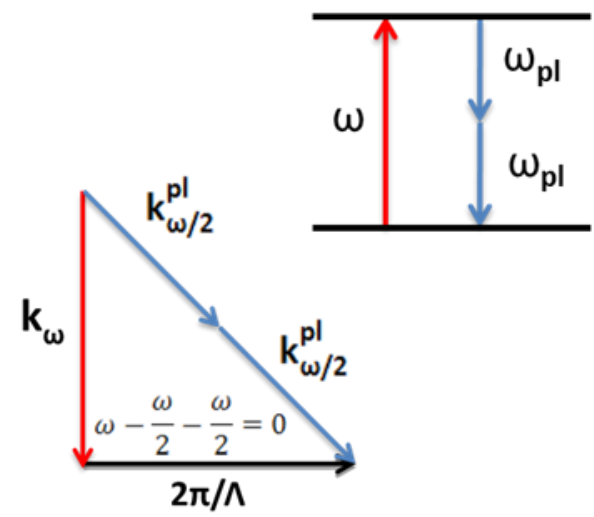

Fig. 2 a) 3/2 harmonic dependence on the degree of linear polarization of the pump beam. A quarter-wave plate was rotated with respect to the input polarization, changing it from linear to circular polarization ( 1 representing linear polarization and 0 for circular polarization). i) Pump beam is linearly polarized and generation of (3/2) $\omega$ increases steadily. ii) Signal increases as a polarization of the beam becomes more elliptical. iii) Signal starts decreasing the polarization of pump beam becomes circular. b) Diagram of nanostructure formation mechanism. Incoming photon interferes with the two plasmons generated from decay to generate nanograting of the period $2 \pi / \Lambda$.

The $3 / 2$ harmonic signal was observed to have peculiar dependence on the polarization of the pump beam (Figure 2a). A quarter-wave plate was placed in the pump beam and rotated to continuously change the pump beam's polarization from linear to circular. The signal reaches maximum, which is about three times stronger than for linear polarization, at about 0.4 degree of linear polarization. When the pump becomes more elliptical, the $3 / 2$ harmonic signal starts decreasing to the level similar as for linear polarization. This shows that the polarization of the pump beam has a direct correlation to the harmonics generated and could play an important role in ultrafast laser nanostructure formation. Detailed experimental investigation is currently in progress.

The observed emission of the three halves harmonic clarifies a long standing issue on electron concentration values in ultrafast laser material modification regimes. Since the two plasmon decay is possible for the electron concentration $n_{e}=n_{\text {cr }} / 4[1,2]$, where $n_{\text {cr }}$ is the critical plasma density, we could state electron concentration is at least $4 \times 10^{20} \mathrm{~cm}^{-3}$.

The experimental observations seen provide evidence that plasmons are being excited in the bulk of the material. It is believed the nanostructure formation involves the interference between the pump photon and the two plasmons generated from the parametric decay process (Figure $2 \mathrm{~b}$ ). This interference is the cause of formation of the nanogratings.

\section{References}

[1] Y. Shimotsuma et al, "Self-organized nanogratings in glass irradiated by ultrashort light pulses," Phys. Rev. Lett. 91, 247405 (2003).

[2] V. Bhardwaj et al, "Optically produced arrays of planar nanostructures inside fused silica," Phys. Rev. Lett. 96, 057404 (2006).

[3] L. P. R. Ramirez et al, "Tuning the structural properties of femtosecond-laser-induced nanogratings," Appl. Phys. A 100, 1 (2010).

[4] J. Canning et al, "Anatomy of a femtosecond laser processed silica waveguide," Opt. Mat. Express 1, 998 (2011).

[5] P. G. Kazansky et al, "Self-assembled nanostructures and two-plasmon decay in femtosecond processing of transparent materials," in Conference on Lasers and Electro-Optics/Quantum Electronics, (OSA, 2007), paper CThJ3.

[6] M. Watanabe et al, "Microfabrication by a high fluence femtosecond exposure: mechanism and applications," SPIE. 4637, 159 (2002). [7] P. G. Kazansky and V. Pruneri, "Electrically stimulated light-induced second-harmonic generation in glass: evidence of coherent photoconductivity," Phys. Rev. Lett. 78, 2956 (1997). 